\title{
Harnessing anthocyanin-rich fruit: a visible reporter for tracing virus-induced gene silencing in pepper fruit
}

\author{
Jihyun Kim¹, Minkyu Park ${ }^{1,4}$, Eun Soo Jeong ${ }^{2}$, Je Min Lee ${ }^{1,2^{*}}$ and Doil Choi ${ }^{1,3^{*}}$
}

\begin{abstract}
Background: Virus-induced gene silencing (VIGS) has become a powerful tool for post-genomic technology in plant species. This is important, especially in select plants, such as the pepper plant, that are recalcitrant to Agrobacteriummediated transformation. Although VIGS in plants has been widely employed as a powerful tool for functional genomics, scattering phenotypic effects by uneven gene silencing has been implemented in order to overcome challenges in experiments with fruit tissues.

Results: We improved the VIGS system based on the tobacco rattle virus (TRV) containing the An2 MYB transcription factor, which is the genetic determinant of purple colored- or anthocyanin-rich pepper. Silencing of endogenous An2 in the anthocyanin-rich pepper with the modified TRV vector for ligation-independent cloning (LIC) lacked purple pigment in its leaves, flowers, and fruits. Infection with TRV-LIC containing a tandem construct of An2 and phytoene desaturase (PDS) resulted in a typical photobleaching event in leaves without the purple pigment, whereas silencing of PDS led to the presence of photobleached and purple-colored leaves. Cosilencing of endogenous An2 and capsaicin synthase in fruits resulted in decreased levels of capsaicin and dihydrocapsaicin as assessed by high performance liquid chromatography analysis coupled with the absence of the purple pigment in fruits.
\end{abstract}

Conclusions: VIGS with tandem constructs harboring An2 as a visible reporter in anthocyanin-rich pepper plants can facilitate the application of functional genomics in the study of metabolic pathways and fruit biology.

Keywords: VIGS, Pepper, TRV, Reporter system, An2, Anthocyanin, Capsaicinoid

\section{Background}

Over the last decade, more than 90 plant genomes and a large amount of transcriptome data have been available due to revolutionary advances in next generation sequencing technologies (https:/en.wikipedia.org/ wiki/List_of_sequenced_plant_genomes). Since the advent of the post-genomic era, functional genomics has been increasingly essential in order to identify genes of unknown function. In contrast to the innovative progress in generating sequence data, advances in functional

\footnotetext{
*Correspondence: jemin@knu.ac.kr; doil@snu.ac.kr

${ }^{1}$ Department of Plant Science, Plant Genomics and Breeding Institute,

College of Agriculture and Life Sciences, Seoul National University,

1 Gwanak-ro, Gwanak-gu, Seoul 08826, Korea

${ }^{2}$ Department of Horticultural Science, Kyungpook National University,

80 Daehakro, Bukgu, Daegu 41566, Korea

Full list of author information is available at the end of the article
}

studies of genes are many steps behind. Due to rapid straightforward methods, virus-induced gene silencing (VIGS) has become one of the most widely used tools in plant functional genomics [1, 2].

The pepper is a fleshy fruit-bearing plant and represents a nutritionally and economically important crop as a staple vegetable and food additive. Pepper consumption by humans has increased since it was determined that their fruits synthesize high levels of many health-promoting compounds including capsaicinoids, vitamin $\mathrm{C}$, and carotenoids [3]. Therefore, breeding and biotechnology focused on enhancing the production of these metabolites are in demand although gene repertories regulating the pathways of these compounds are not fully understood. The pepper genome, however, can accelerate the identification of many genes potentially associated with 
important agronomic traits [4]. Pepper is closely related to the tomato plant, which is a model for fleshy fruit biology and biotechnology. Comparative genomics in Solanaceae accelerated the discovery of genes responsible for many agronomically important traits $[4,5]$. Unlike tomato and other Solanaceae plants, pepper is recalcitrant to Agrobacterium-mediated-transformation; thus, VIGS has proven to be a powerful method for determining the function of unknown genes. The TRV system was widely employed for gene silencing in Solanaceae, including pepper plants [6-8]. These previous experiments were limited to vegetative tissues, which led to difficulties in the interpretation of the effects of virus and gene expression in the late stages of fruit development. Therefore, it is essential to develop molecular tools for the functional analysis of gene expression in pepper fruits responsible for biochemical processes of human healthpromoting metabolites.

Although VIGS in plants has being widely employed as a powerful tool for functional genomics, scattering phenotypic effects driven by uneven gene silencing has become a problem that must be overcome, especially in fruit tissue. To ameliorate this limitation of VIGS, visual reporter systems were developed using GFP [9] and DEL-ROS [10] in GFP overexpressing and anthocyaninenriched transgenic plants, respectively. Since silenced areas were visually traceable in these systems, dissection and sampling of tissues for subsequent metabolic analysis successfully identified gene functions impacting the metabolites of interest [9-11].

In this study, we improved the VIGS system to monitor gene silencing in pepper fruit. For this purpose, we employed the TRV-LIC VIGS system [12] for highthroughput cloning and used $A n 2$ [13] as a reporter, which is the genetic determinant of purple pigmentation due to the accumulation of anthocyanin in pepper. We successfully examined the cosilencing effects of $P D S$ and capsaicin synthase coupled with $A n 2$, and the subsequent phenotypic and metabolic changes in leaves and fruits, respectively. Our results advance reverse genetics methods for fruit traits and ultimately enhance the understanding of the molecular mechanisms of novel genes and regulators in fruit-specific metabolic pathways.

\section{Methods}

\section{Plant materials and growing conditions}

Capsicum annuum cv. NuMex Halloween (hereafter $\mathrm{NMH}$ ), kindly provided by Prof. Byoung-Cheorl Kang (Seoul National University), was used in this study and maintained in growth chambers. Pungent $\mathrm{NMH}$ contained visible amounts of anthocyanin in leaf, stem, flower, and fruit. The plant height was approximately
$30 \mathrm{~cm}$. After germination on plates at $30^{\circ} \mathrm{C}$, the seedlings were transferred to plug trays at $25^{\circ} \mathrm{C}$ with a $16 / 8 \mathrm{~h}$ light/ dark photoperiod until cotyledons were fully expanded (approximately 2 weeks after germination). After agroinfiltration to the abaxial side of the cotyledons, pepper plants were incubated at $16{ }^{\circ} \mathrm{C}$ under dark conditions for 1 day. Four-week-post-infiltrated plants were transplanted to pots $(130 / 115 \mathrm{~mm})$ and grown at $20{ }^{\circ} \mathrm{C}$ with a $16 / 8 \mathrm{~h}$ light/dark photoperiod. The plants were fertilized by WUXAL according to the manufacturer's instructions (WUXAL calcium, AGLUKON, Germany) once every 2 months.

\section{Plasmid construction}

The TRV vectors, pTRV1 [14] and pTRV2-LIC [12], were kindly provided by Dr. Dinesh Kumar, at UC Davis. The ligation independent cloning (LIC) was conducted as described in Dong et al. [12] and Fig. 1. For gene cloning in pTRV2-LIC, the gene of interest (GOI) was amplified with primers: 5'-CGACGACAAGACCCT (LIC vector adaptor)-gene specific sequences- $3^{\prime}$ and $5^{\prime}$-CTTTGTCTAGTG ( $A n 2$ adaptor)-gene specific sequences- $3^{\prime}$ using SolgTM $P f u-X$ DNA polymerase (Solgent, Korea). An2 was amplified with primers An2_F and An2_lic_R (Table 1). Fragments of PDS (CA03g36860, $173 \mathrm{bp}), C S$ (CA02g19260, $181 \mathrm{bp}$ ), and An2 (CA10g11650, $258 \mathrm{bp})$ were amplified from the pepper leaf, placenta, and pericarp cDNA, respectively. TRV2-GFP was previously used for VIGS as a control [15]. The insert size of each target gene and $A n 2$ was $150-300 \mathrm{bp}$ and the insert size of fused cDNAs was 400-600 bp in the TRV2-LIC vector. Using the amplified cDNAs of the target genes and $A n 2$ as template, PCR was performed to fuse both cDNA fragments in the sense orientation. The PCR products were purified with DNA Clean and Concentrator ${ }^{\mathrm{TM}}$ (Zymo Research, USA). A total 100 ng of purified PCR product was treated with T4 DNA polymerase (New England Biolabs, USA) in $1 \times$ reaction buffer containing $10 \mathrm{mM}$ dATP and dithiothreitol at $22{ }^{\circ} \mathrm{C}$ for $30 \mathrm{~min}$ followed by 20 min of inactivation of T4 DNA polymerase at $70{ }^{\circ} \mathrm{C}$. The TRV2-LIC vector was digested by the restriction enzyme Pst $\mathrm{I}$ and treated with T4 DNA polymerase and dTTP instead of dATP. A total of $50 \mathrm{ng}$ of PCR product and TRV2-LIC vector were mixed and incubated at $65{ }^{\circ} \mathrm{C}$ for $1 \mathrm{~min}$ and then $22{ }^{\circ} \mathrm{C}$ for $10 \mathrm{~min}$. The mixture was transformed into E. coli $\mathrm{DH} 10 \mathrm{~B}$ or DH5 $\alpha$ competent cells. Transformants were selected by PCR using primers for sequencing (Table 1) and confirmed by DNA sequencing. The plasmids from the transformants were introduced into Agrobacterium tumefaciens strain GV3101 using the freeze-thaw method [16]. 


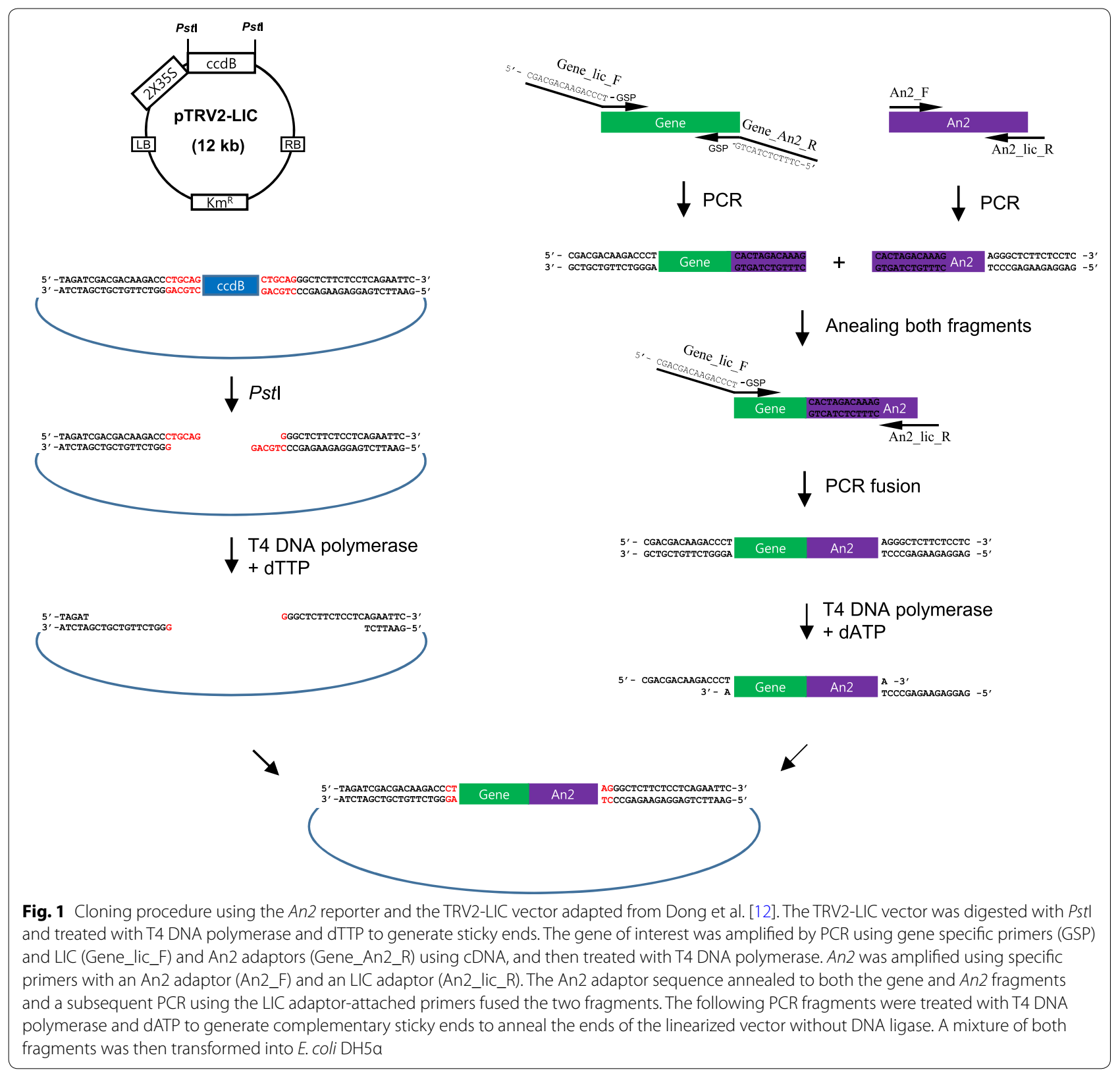

\section{Agroinfiltration}

Agrobacterium tumefaciens strain GV3101 carrying pTRV1, pTRV2::GFP, pTRV2-LIC::GFP::An2, pTRV2LIC::PDS::An2, pTRV2-LIC::CS::An2, and was grown overnight at $28{ }^{\circ} \mathrm{C}$ in $10 \mathrm{~mL}$ YEP medium containing rifampicin $(50 \mu \mathrm{g} / \mathrm{mL})$ and kanamycin $(50 \mu \mathrm{g} / \mathrm{mL})$ as described [6]. The transformed Agrobacterium was harvested by centrifugation at $13,000 \times g$ for $15 \mathrm{~min}$ at $20^{\circ} \mathrm{C}$, and resuspended in $10 \mathrm{mM} \mathrm{MES,} 10 \mathrm{mM} \mathrm{MgCl}$, and $200 \mu \mathrm{M}$ acetosyringone to a final $\mathrm{OD}_{600} \mathrm{~nm}$ of 0.7 . Cell suspensions were incubated at room temperature with inverting for $4 \mathrm{~h}$. Agrobacterium cultures containing
pTRV1 and pTRV2-LIC carrying any GOI were mixed at a 1:1 ratio and infiltrated into the abaxial side of the both cotyledons. Leaves from 4-week-old plants and placenta from fruits from plants 30 days post-anthesis (DPA) were harvested and immediately frozen in liquid nitrogen for qRT-PCR and HPLC. Due to a high frequency of fruit abscission, at least five silenced plants per each construct were grown at $20^{\circ} \mathrm{C}$ until harvest.

\section{RNA isolation and quantitative RT-PCR analysis}

The total RNA from $100 \mathrm{mg}$ of tissue was extracted using the TRIzol ${ }^{\circledR}$ reagent (Invitrogen, USA). Total RNA $(5 \mu \mathrm{g})$ 
Table 1 List of primer sequences used in this study

\begin{tabular}{|c|c|}
\hline Primer & Sequence $\left(5^{\prime}-3^{\prime}\right)$ \\
\hline \multicolumn{2}{|l|}{ VIGS construct } \\
\hline CS_lic_F & CGACGACAAGACCCTGAGAAGGGAAACTGCCATTTGA \\
\hline CS_An2_R & CTTTGTCTAGTG CCTTGCCCAGCTTTGTAATCTT \\
\hline GFP_lic_F & CGACGACAAGACCCTCACGGCAGACAAACAAAAGA \\
\hline GFP_An2_R & CTTTGTCTAGTG AAAGGGCAGATTGTGTGGAC \\
\hline An2_F & CACTAGACAAAGACGAACGCGAC \\
\hline An2_lic_R & GAGGAGAAGAGCCCTCAGAAAAGTCATCCCAACCATCAC \\
\hline PDS_lic_F & CGACGACAAGACCCTCTTGCAAAGATCCCCTGTAG \\
\hline PDS_An2_R & CTTTGTCTAGTGCACTTGTTTCTGCCAACTTC \\
\hline \multicolumn{2}{|l|}{ Sequencing } \\
\hline TRV2_seq_F & CTGTTTGAGGGAAAAGTAG \\
\hline TRV2_seq_R & CAAAAGACTTACCGATCAATC \\
\hline \multicolumn{2}{|l|}{ QRT-PCR } \\
\hline CS_F & TTCCCATATAGCCCACTTGC \\
\hline CS_R & ACTACAAGCAAATTACCACCTTC \\
\hline PDS_F & AGCAAAGCAAAAATATTGAAGTA \\
\hline PDS_R & GCTTTCCTGATAAGACAGC \\
\hline$A n 2 \_F$ & GGAGAAGGAAAGTGGCATCTTGT \\
\hline An2_R & САCCTCTCTTTATATGCGGCCTT \\
\hline CaActin_F & ATGGCAGATGAAGATATTCAAC \\
\hline CaActin_R & ACTAGGAAAAACAGCCCTTGGT \\
\hline
\end{tabular}

was reverse-transcribed with Oligo (dT) primers and Superscript II (Invitrogen), according to the manufacturer's instructions. Subsequently, qRT-PCR was conducted to analyze gene expression level using the SYBR Green PCR master mix (Invitrogen, USA) and genespecific primers (Table 1) in the Rotor-Gene 6000 apparatus (QIAGEN, USA), according to the manufacturer's instructions. All statistical analyses were conducted as described in the manufacturer's protocol. To normalize the expression levels, the transcript level of CaActin (CA00g80270) was used as a control. Duplicates from 3 biological replicates were used in the qRT-PCR analysis.

\section{Capsaicinoid extraction and HPLC analysis}

The placental tissue of each fruit at 30 days-post anthesis was isolated and immediately frozen in liquid nitrogen. For sampling in An2-silenced plants, purple pigmentdepleted placenta was carefully collected. Previous method of capsaicinoid extraction [17] was modified for frozen tissues. Approximately $100 \mathrm{mg}$ of frozen powder from pooled samples of two fruits was extracted with $1.5 \mathrm{~mL}$ of an ethyl acetate and acetone mixture (6:4) using TissueLyser II (QIAGEN, USA) at room temperature for $10 \mathrm{~min}$ and incubated with shaking at $37^{\circ} \mathrm{C}$ for $1 \mathrm{~h}$. After centrifugation at $12,000 \times g$ for $5 \mathrm{~min}$ at room temperature, $600 \mu \mathrm{L}$ of the supernatant was transferred to a new tube and evaporated in an Automatic Environmental
SpeedVac System AES1010 (Operon, Korea). The extract was dissolved in $500 \mu \mathrm{L}$ methanol and filtered using an Acrodisc $^{\circledR}$ LC $13-\mathrm{mm}$ syringe filter with a $0.2-\mu \mathrm{m}$ PVDF membrane (Sigma-Aldrich, USA). Capsaicinoid analysis was performed using the UltiMate ${ }^{\circledR} 3000$ HPLC (Dionex, USA) including the Inno C-18 column (4.6 mm $\times 150 \mathrm{~mm}$, Young)in Biochrom, Korea). A UV detector was operated at $280 \mathrm{~nm}$ and the data acquisition was performed with Chromeleon software. Separation of capsaicinoids was achieved under $75 \% \mathrm{MeOH}$ at a flow rate of $1 \mathrm{~mL} / \mathrm{min}$. Each $10 \mu \mathrm{L}$ aliquot was analyzed with HPLC. The HPLC analyses were performed at NICEM, Seoul National University. Capsaicin and dihydrocapsaicin used as standard compounds were purchased from Sigma-Aldrich (M2028 and M1022, respectively) [17].

\section{Results}

Improvement of VIGS in the pepper plant using a TRV2-LIC system and An2

The purpose of this study was to develop efficient gene silencing in pepper fruit based on the TRV2-LIC system [12]. The TRV2-LIC system utilizes the exonuclease activity of T4 DNA polymerase to generate sticky ends in both the insert and the vector (Fig. 1). Then, $c c d B$ in the vector was used to efficiently select putative recombinant colonies. Therefore, the TRV2-LIC system enabled us to clone any gene of interest (GOI) in a high-throughput manner. Although TRV systems were successfully used previously in Solanaceae, VIGS in pepper fruits has not been fully investigated. To improve silencing efficiency and uniformity in fruit, we used $A n 2$ as a reporter gene in the TRV2-LIC construction to visualize the sector subject to silencing in the purple pepper. The $A$ locus controls anthocyanin accumulation in various tissues of the pepper plant [18] and the R2R3-MYB transcription factor, $A n 2$, has been identified as the candidate gene of the $A$ locus [13]. The expression of $A n 2$ was detected in all stages of fruit development and also in both the flower and leaf, resulting in anthocyanin accumulation. To verify the function of $A n 2$ and its possible use as a reporter for VIGS, a gene specific fragment of $A n 2$ (CA10g11650, $258 \mathrm{bp}$ ) was incorporated into the TRV2-LIC vector (Figs. 1, 2b).

\section{Silencing and its assessment of endogenous An2 in different tissues}

Agrobacterium carrying TRV2::GFP as a control and TRV2::GFP::An2 with TRV1 were infiltrated in the cotyledons of 2-week-old seedlings of NMH to extend the silencing response to meristematic tissues (Fig. 2a). GFP was used for control of gene fragment and the GFP gene fragment is not existed in the pepper genome. Therefore, GFP in the TRV2-LIC would not affect any gene 

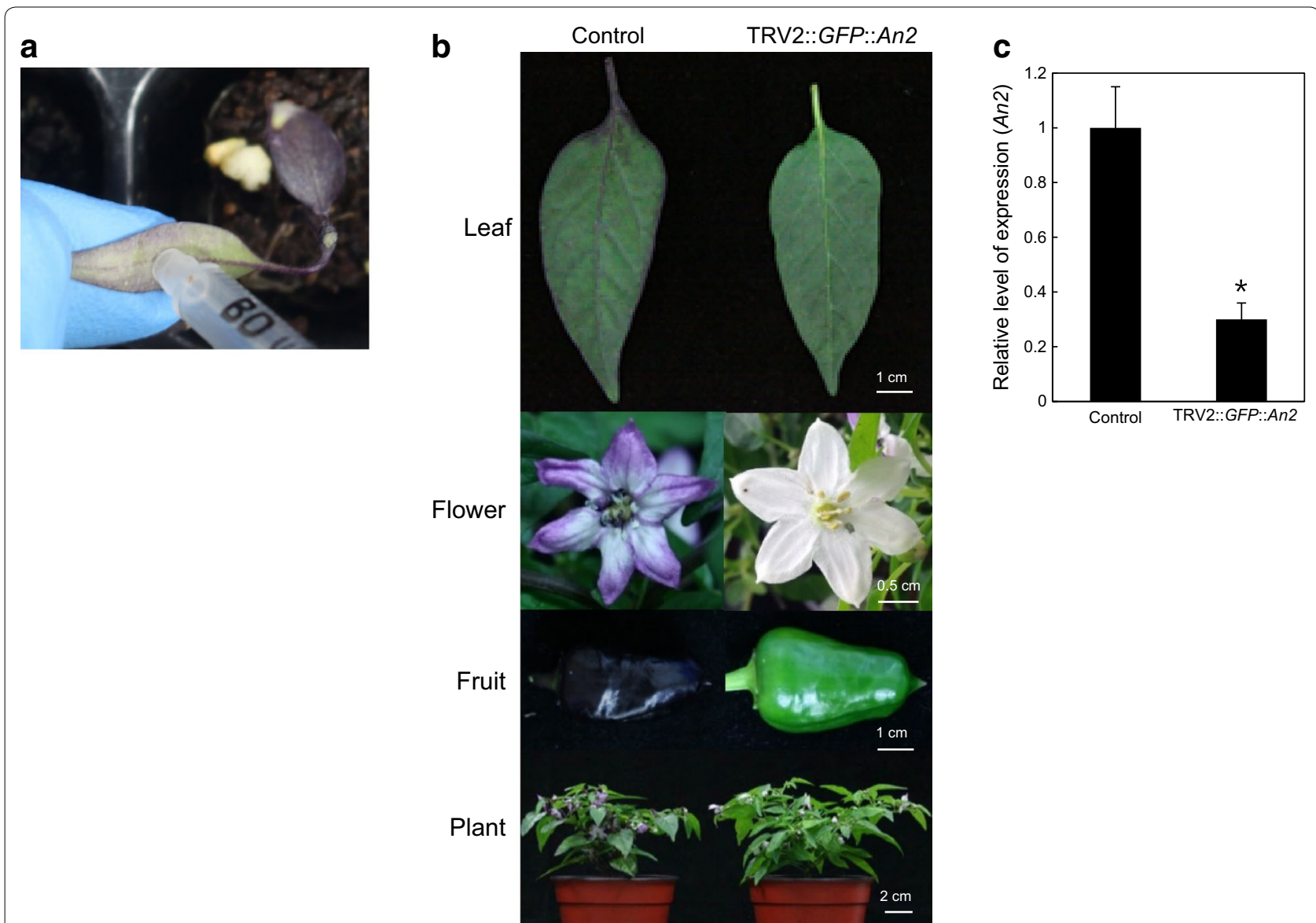

Fig. 2 Anthocyanin-mediated visualization of VIGS in peppers using TRV2-LIC and An2 as a reporter. a Agroinfiltration to NMH cotyledons. b Results from different tissues of TRV2-GFP-infiltrated pepper (control, left) and TRV2::GFP::An2 infiltrated pepper (right). c Expression analysis of An2 by qRTPCR in 30 DPA fruits. Data indicate relative expression level compared to control. Three biological replicates of qRT-PCRs were performed. Data are shown as mean \pm SE. Statistically significant differences, determined by Student's t test, are indicated by an asterisk $(P \leq 0.05)$

expression or phenotype. The infiltrated plants were grown at $20{ }^{\circ} \mathrm{C}$ to maintain silencing for a long period and enhance silencing efficiency. NMH, dwarf, and ornamental pepper cultivars, were characterized by purple leaf pigmentation, flowers, and immature fruits, and harbored orange colored ripe fruits. Fruits were oriented upright, were bullet shaped, and pungent (http://www. chilepepperinstitute.org/cart/product/100/numex_halloween/). Figure $2 \mathrm{~b}$ demonstrates the phenotypes of the control and An2-silenced peppers in different tissues. $A n 2$ silencing using TRV2-LIC systems clearly resulted in anthocyanin deficiency in leaves, flowers, and fruits. In flowers of $A n 2$-silenced plants, the absence of the purple pigment was also observed in the stigma and stamen. To examine whether the fruit phenotypes observed in the infiltrated peppers were associated with the downregulation of $A n 2$, qRT-PCR was conducted to compare the gene expression between purple and green fruits. The level of $A n 2$ expression was remarkably decreased in
TRV2::GFP::An2 compared to the control (Fig. 2c), and the impact of $A n 2$ silencing was negligible compared to the control. Therefore, $A n 2$ was chosen as a reporter in this VIGS system for NMH by monitoring anthocyanin accumulation. These results also indicate that $A n 2$ is the genetic determinant of the $A$ locus [13].

\section{Validating the cosilencing method in the tandem construct using PDS and An2}

To validate the cosilencing effect of $A n 2$ and GOI, the prevention of purple pigmentation by $A n 2$ silencing and subsequent phenotypic analysis by GOI silencing were investigated. As a proof of concept, we employed PDS to verify that cosilencing occurred with $A n 2$ in NMH. A gene specific fragment of PDS (CA03g36860, $173 \mathrm{bp}$ ) was tandemly fused with $A n 2$ in the TRV2-LIC system and this construct was then agroinfiltrated in the cotyledons of NMH (Fig. 1). Subsequently, PDS and An2 mRNA levels were measured using qRT-PCR in 4-week-old leaves. 
Silencing of PDS resulted in photobleaching of $\mathrm{NMH}$ leaves and did not prevent anthocyanin pigmentation. However, cosilencing of PDS and An2 was shown to cause photobleaching coupled with a lack of anthocyanin in leaves of NMH (Fig. 3). There were no significant differences in the levels of $P D S$ mRNA between TRV2::PDS and TRV2::PDS::An2 leaves, suggesting silencing of $A n 2$ did not affect $P D S$ expression. The expression level of $P D S$ significantly decreased in both TRV2::PDS and TRV2::PDS::An2 leaves. However, An2 expression notably decreased only in TRV2::PDS::An2 leaves.

\section{Validating cosilencing in fruits using capsaicin synthase and $A n 2$}

Capsaicinoids are specialized metabolic determinants of pungency and natural vanilloid, which are only found in pepper species. Capsaicin synthase (CS), a homologue of acyltransferase, is the genetic factor of the $C$ or Pun1 locus controlling pungency [19]. Capsaicin synthase catalyzes the last step of the pathway by condensing vanillylamine to 8-methyl-6-nonenoyl-CoA [20]. Capsaicinoids are mostly synthesized in placenta tissue and capsaicin synthases are only expressed during pepper placenta development [19].

To validate the cosilencing effect of GOI with $A n 2$ in fruits and its potential use as a tool for metabolic genetics, a partial cDNA of CS (CA02g19260, 181 bp) was tandemly fused with $A n 2$ in the TRV2-LIC system and this construct was agroinfiltrated as described above. To maintain and allow for the VIGS signal to reach fruits, the agroinfiltrated peppers were grown at $20{ }^{\circ} \mathrm{C}$ until harvest. Due to the spatiotemporal regulation of capsaicinoid biosynthesis and $C S$ expression, placenta tissues of at least six different 30 DPA fruits were collected for further quantification of capsaicinoid and CS transcripts. The visual phenotypes of representative fruits, resulting quantification of $C S$ transcripts as well as the capsaicinoids are shown in Fig. 3. In the control, anthocyanin mainly accumulated in the exocarp (not the endocarp) and was clearly visible in placenta tissues (Fig. 4a). Silencing of $A n 2$ resulted in the loss of anthocyanin pigmentation in the exocarp and placenta of TRV2::GFP::An2 and TRV2::CS::An2. To assess the cosilencing effects of CS and $A n 2$ in the placenta, the anthocyanin-depleted placenta (30 DPA) was carefully dissected and used for further analysis with qRT-PCR and HPLC. There were no significant differences in the levels of CS mRNA between the control (TRV2::GFP) and TRV2::GFP::An2 leaves, suggesting that silencing of $A n 2$ did not affect $C S$ expression. The expression levels of $C S$ were significantly lower in TRV2::CS::An2 than in the control or TRV2::GFP::An2 (Fig. 4b). To address whether $C S$ silencing affected capsaicinoid accumulation in the placenta, capsaicinoids were quantified in the same tissues used for qRT-PCR.
The HPLC method was able to identify biosynthesis of capsaicin and dihydrocapsaicin, which are major the capsaicinoids in pepper fruits. Thus, $A n 2$ silencing did not affect capsaicinoid content. However, $C S$-silenced plants showed a significant $50 \%$ reduction of capsaicin and dihydrocapsacin, as expected, in 30 DPA-placenta (almost $50 \%)$ compared to the control and TRV::GFP::An2 (Fig. 4c). Any other phenotypic changes caused by cosilencing $C S$ and $A n 2$ was not observed compared to the $A n 2$-silenced plant, suggesting that these systems are feasible methods to use in functional plant genomics. Metabolic phenotypes of the silenced fruits were in agreement with enzyme function. The anthocyanin accumulation or depletion in silenced placenta tissues had no significant effect on capsaicinoid biosynthesis or expression of $C S$.

\section{Discussion}

A reporter gene is required in VIGS to visualize the silenced region and monitor the silencing efficiency. PDS has been widely used as a reporter of choice [21]. Since $P D S$ silencing decreased chlorophyll and carotenoid biosynthesis resulting reduced photosynthetic activity, $P D S$ silenced plant showed growth defect [22]. PDS silencing also caused carotenoid-deficient fruits and affected other metabolisms beyond carotenoid in tomato fruits [10, 23]. Thus, use of $P D S$ as a reporter expect to show poor fruit set and disturb important metabolisms in pepper fruits. Unlike $P D S, A n 2$ silencing did not affect other phenotype except anthocyanin pigmentation. Therefore, we choose $A n 2$ as a reporter in this study.

Despite the advantages and utility of VIGS in plant functional genomics studies [1, 2], the uneven distribution of silencing in target tissues is a major limitation. Use of a visible reporter would lead to the efficient sampling of silenced tissues from nonsilenced tissues, therefore increasing the sensitivity of subsequent analysis. Several studies have successfully overcome this limitation using transgenic plants of DEL-ROS $[1,10,11]$ and GFP [9]. Using the transgenes as visible reporters, cosilencing with the reporter and GOI enables us to identify the precise region where silencing occurs. Ectopic expression of transcription factors, $D E L$ and ROS1 using a ripe-fruit specific promoter resulted in the up-regulation of the anthocyanin biosynthetic genes, leading to anthocyanin accumulation in ripening tomato fruits. Cosilencing the reporter genes with GOI in the transgenic plants facilitated visualization of the silenced region. Fluorescence of the GFP-silenced region in GFP-overproducing plants was clearly decreased after exposure to UV. Therefore, target tissues can be easily collected and the effect of silencing can be more easily identified in systems with higher phenotypic variation compared to the non-collective method. However, these methods are still limited in 


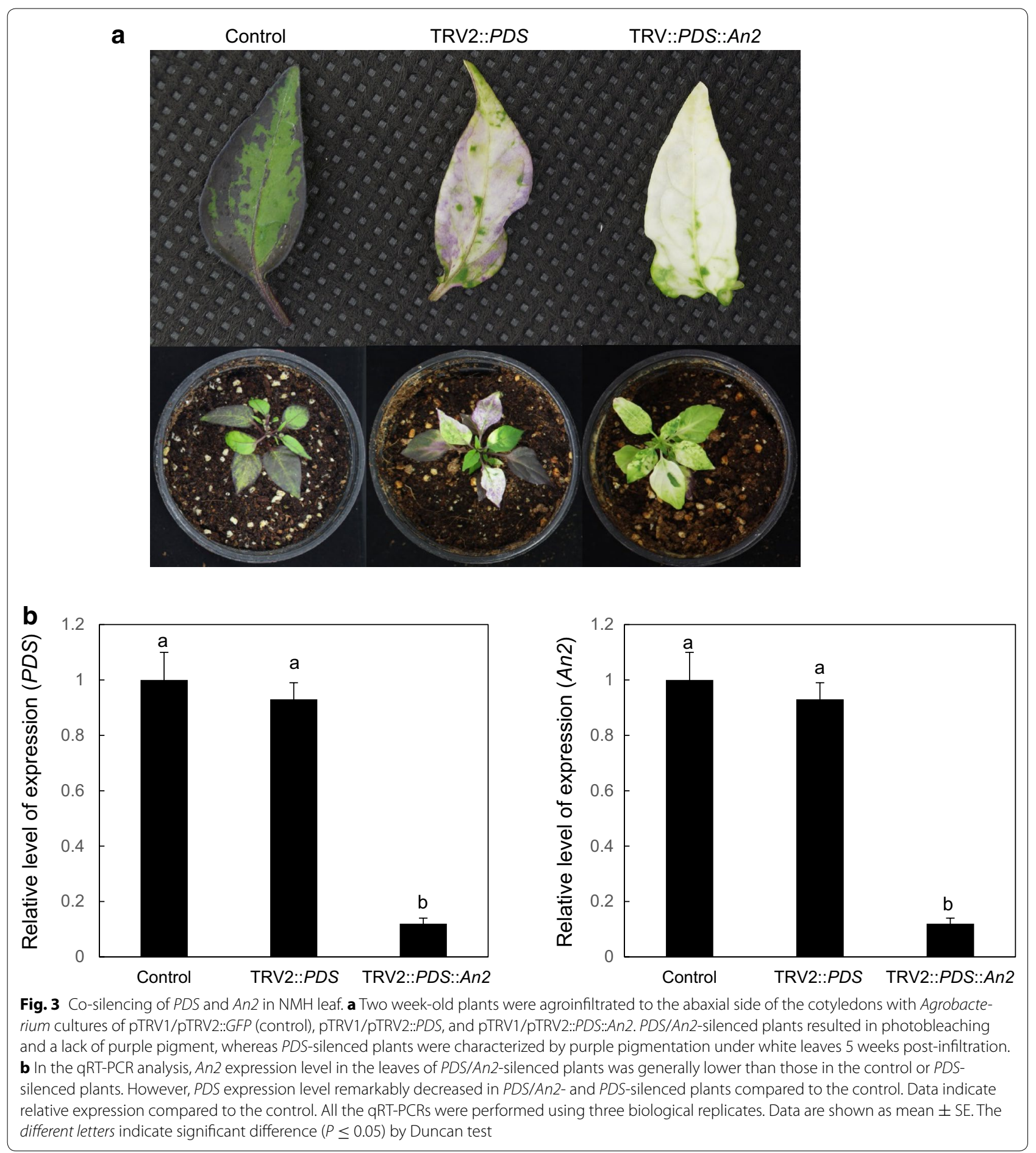

plant species, which are recalcitrant to Agrobacteriummediated transformation. In order to extend this concept to most the majority of plant species, we established the endogenous reporter system, $A n 2$, under natural variation of anthocyanin-rich pepper. Although we intended to develop this system for fruit genetics, the reporter in
$\mathrm{NMH}$ can easily be used to monitor the silenced sector of genes in leaves and flowers and facilitate collection of tissues without further expression analysis of GOI, as we revealed that it was possible to cosilence $P D S$ and $A n 2$ (Fig. 3). Therefore, our results will be helpful to study genes controlling diverse biological phenomena. Previous 
studies on CS knockdown by VIGS showed reduced capsaicinoid content in fruits, which is in agreement with the results of our study (Fig. 4) [19, 24], clearly indicating that $C S$ is responsible for capsaicinoid synthesis. CS, originally referred to as Pun1, is believed to catalyze the last step of capsaicinoid biosynthesis although there is no direct evidence that Pun1 has capsaicin synthase activity. Recently, using a protoplast-based assay for de novo capsaicin synthesis and antibodies of the anti-Pun1, which are antagonists of endogenous Pun1 activity, the Pun1 gene and its product were proven to be involved in capsaicin synthesis [24]. These data together with our results reveal that CS or Pun1 primarily controls the final step in capsaicinoid biosynthesis.
VIGS using TRV systems efficiently functioned in vegetative pepper tissues [6]. However, the silencing signal is not well transmitted throughout the whole plant, particularly in reproductive tissues. Silencing of $P D S$ was previously maintained in flowers and tomato fruits and enhanced by low temperature and low humidity [25]. In our study, low temperature was a critical requirement of VIGS in fruits, although fruits were often characterized by evidence of abscission before harvest (data not shown). Further improvement using different environmental conditions and diverse pepper cultivars should be thoroughly examined. In addition, $A n 2$ as a transcription factor, should not dramatically affect the developmental or biochemical regulation
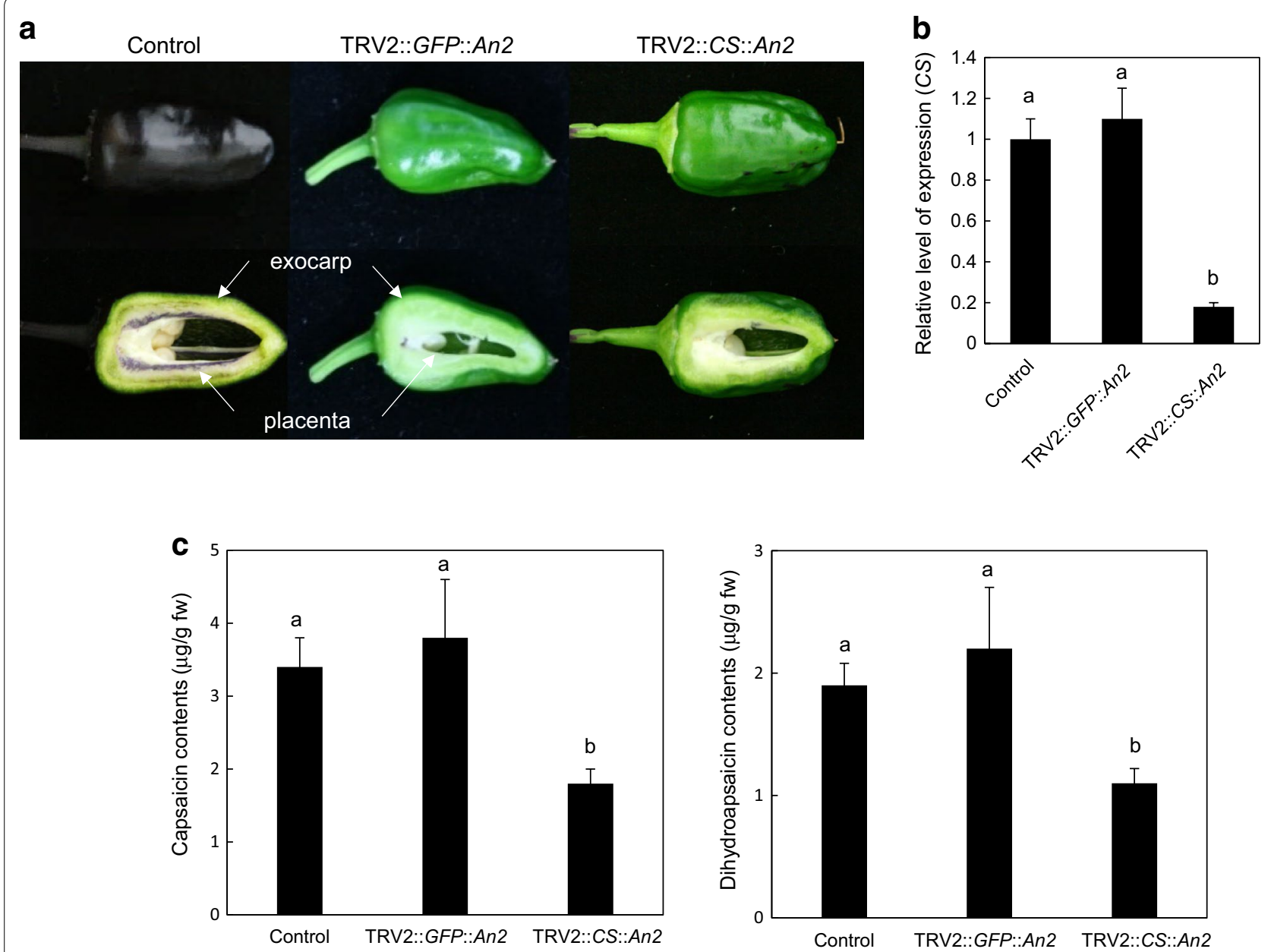

Fig. 4 Cosilencing of capsaicin synthase (CS) and An2. a Anthocyanin-mediated visualization of cosilencing of CS and An2 in pepper pericarp and placenta (30 DPA). Silencing of An2 resulted in the depletion of anthocyanin pigmentation in the exocarp and placenta of TRV2::GFP::An2 and TRV2::CS::An2. b Gene expression analysis of CS in the cosilenced fruits (placenta) compared to the control and An2-silenced fruits by qRT-PCR. The expression levels of CS were significantly lower in TRV2::CS::An2 than in the control and TRV2::GFP::An2. c Capsaicinoid analysis in the cosilenced fruits compared to control and An2-silenced fruits by HPLC $(n=6)$. Cosilencing of An2 and CS in the placenta resulted in decreased levels of capsaicin and dihydrocapsaicin. Asterisks indicate significant differences $(P \leq 0.05)$ between TRV2::GFP::An2 and TRV2::CS::An2. Data are shown as mean \pm SE. The different letters indicate significant difference $(P \leq 0.05)$ by Duncan test 
underlying the function under study, for example, in cases when the GOI is a transcription factor or other master regulator.

\section{Conclusions}

Here, we present the utility of the gene silencing approach, which uses an endogenous reporter, $A n 2$, in anthocyanin-rich peppers, both by down-regulating $P D S$ in leaves and $C S$ in fruits, ultimately demonstrating the feasibility of this system in metabolic genetics in pepper fruits. Our results demonstrate the power of this tool for addressing the roles of regulatory genes in capsaicinoid biosynthesis and secondary metabolism. The application of this system to examine candidate regulatory genes will be useful and facilitate confirmation of gene functions identified in the model species: tomato and Arabidopsis.

\section{Abbreviations}

VIGS: virus-induced gene silencing; TRV: tobacco rattle virus; GOI: gene of interest; LIC: ligation independent cloning; PDS: phytoene desaturase; CS: capsaicin synthase; HPLC: high performance liquid chromatography; DPA: days post-anthesis.

\section{Authors' contributions}

The study was conceived and designed by JK, MK, JML, and DC, experiments were performed by JK, ESJ, and JML, and the manuscript was written by JK, $J M L$, and DC. All authors read and approved the final manuscript.

\section{Author details}

${ }^{1}$ Department of Plant Science, Plant Genomics and Breeding Institute, College of Agriculture and Life Sciences, Seoul National University, 1 Gwanak-ro, Gwanak-gu, Seoul 08826, Korea. ${ }^{2}$ Department of Horticultural Science, Kyungpook National University, 80 Daehakro, Bukgu, Daegu 41566, Korea. ${ }^{3}$ Crop Biotechnology Institute/GreenBio Science and Technology, Seoul National University, Pyeongchang, Korea. ${ }^{4}$ Present Address: Department of Genetics, University of Georgia, Athens, GA 30602-7223, USA.

\section{Acknowledgements}

This research was supported by the National Research Foundation of Korea (No. 2014R1A1A1038130) to JML and the Vegetable Breeding Research Center (710001-07) through the Ministry of Agriculture, Food and Rural Affairs of the Korean government to DC. We would like to thank Hyun Jung Kim for technical assistance.

\section{Competing interests}

The authors declare that they have no competing interests.

\section{Availability of data and materials}

All the data is contained within the manuscript

\section{Consent for publication}

All the authors have approved the manuscript and have made all required statements and declarations.

\section{Funding}

JML is supported by the National Research Foundation of Korea (No. 2014R1A1A1038130). DC is supported by the Vegetable Breeding Research Center (710001-07) through the Ministry of Agriculture, Food and Rural Affairs of Korea.

Received: 27 August 2016 Accepted: 24 November 2016

Published online: 03 January 2017
References

1. Becker A, Lange M. VIGS-genomics goes functional. Trends Plant Sci. 2010;15:1-4.

2. Senthil-Kumar M, Mysore KS. New dimensions for VIGS in plant functional genomics. Trends Plant Sci. 2011;16:656-65.

3. Wahyuni Y, Ballester AR, Sudarmonowati E, Bino RJ, Bovy AG. Secondary metabolites of Capsicum species and their importance in the human diet. J Nat Prod. 2013;76:783-93.

4. Kim S, Park M, Yeom SI, Kim YM, Lee JM, Lee HA, et al. Genome sequence of the hot pepper provides insights into the evolution of pungency in Capsicum species. Nat Genet. 2014:46:270-8.

5. Paran I, van der Knaap E. Genetic and molecular regulation of fruit and plant domestication traits in tomato and pepper. J Exp Bot. 2007; 58:3841-52.

6. Chung E, Seong E, Kim YC, Chung EJ, Oh SK, Lee S, Park JM, Joung YH, Choi D. A method of high frequency virus-induced gene silencing in chili pepper (Capsicum annuum L. cv. Bukang). Mol Cells. 2004;17:377-80.

7. del Rosario Abraham-Juarez M, del Carmen Rocha-Granados M, Lopez MG, Rivera-Bustamante RF, Ochoa-Alejo N. Virus-induced silencing of Comt, pAmt and Kas genes results in a reduction of capsaicinoid accumulation in chili pepper fruits. Planta. 2008;227:681-95.

8. Tian SL, Li L, Chai WG, Shah SN, Gong ZH. Effects of silencing key genes in the capsanthin biosynthetic pathway on fruit color of detached pepper fruits. BMC Plant Biol. 2014;14:314.

9. Quadrana L, Rodriguez MC, Lopez M, Bermudez L, Nunes-Nesi A, Fernie $A R$, et al. Coupling virus-induced gene silencing to exogenous green fluorescence protein expression provides a highly efficient system for functional genomics in Arabidopsis and across all stages of tomato fruit development. Plant Physiol. 2011;156:1278-91.

10. Orzaez D, Medina A, Torre S, Fernandez-Moreno JP, Rambla JL, FernandezDel-Carmen A, Butelli E, Martin C, Granell A. A visual reporter system for virus-induced gene silencing in tomato fruit based on anthocyanin accumulation. Plant Physiol. 2009;150:1122-34.

11. Fantini E, Falcone G, Frusciante S, Giliberto L, Giuliano G. Dissection of tomato lycopene biosynthesis through virus-induced gene silencing. Plant Physiol. 2013;163:986-98.

12. Dong Y, Burch-Smith TM, Liu Y, Mamillapalli P, Dinesh-Kumar SP. A ligationindependent cloning tobacco rattle virus vector for high-throughput virus-induced gene silencing identifies roles for NbMADS4-1 and -2 in floral development. Plant Physiol. 2007;145:1161-70.

13. Borovsky Y, Oren-Shamir M, Ovadia R, De Jong W, Paran I. The a locus that controls anthocyanin accumulation in pepper encodes a MYB transcription factor homologous to Anthocyanin2 of Petunia. Theor Appl Genet. 2004; 109:23-9.

14. Liu Y, Schiff M, Marathe R, Dinesh-Kumar SP. Tobacco Rar1, EDS1 and NPR1/NIM1 like genes are required for N-mediated resistance to tobacco mosaic virus. Plant J. 2002;30:415-29.

15. Sarowar S, Oh HW, Cho HS, Baek KH, Seong ES, Joung YH, Choi GJ, Lee S, Choi D. Capsicum annuum CCR4-associated factor CaCAF1 is necessary for plant development and defence response. Plant J. 2007;51:792-802.

16. Chen $\mathrm{H}$, Nelson RS, Sherwood JL. Enhanced recovery of transformants of Agrobacterium tumefaciens after freeze-thaw transformation and drug selection. Biotechniques. 1994;16(664-668):670.

17. Han K, Jeong HJ, Sung J, Keum YS, Cho MC, Kim JH, Kwon JK, Kim BD, Kang BC. Biosynthesis of capsinoid is controlled by the Pun 1 locus in pepper. Mol Breed. 2013;31:537-48.

18. Daskalov S, Poulos JM. Updated Capsicum gene list. Capsicum Eggplant Nswl. 1994;13:16-26.

19. Stewart C Jr, Kang BC, Liu K, Mazourek M, Moore SL, Yoo EY, Kim BD, Paran I, Jahn MM. The Pun1 gene for pungency in pepper encodes a putative acyltransferase. Plant J. 2005;42:675-88.

20. Mazourek M, Pujar A, Borovsky Y, Paran I, Mueller L, Jahn MM. A dynamic interface for capsaicinoid systems biology. Plant Physiol. 2009;150:1806-21.

21. Ruiz MT, Voinnet O, Baulcombe DC. Initiation and maintenance of virusinduced gene silencing. Plant Cell. 1998;10:937-46.

22. Parent JS, Bouteiller N, Elmayan T, Vaucheret $H$. Respective contributions of arabidopsis DCL2 and DCL4 to RNA silencing. Plant J. 2015;81:223-32.

23. Romero I, Tikunov Y, Bovy A. Virus-induced gene silencing in detached tomatoes and biochemical effects of phytoene desaturase gene silencing. J Plant Physiol. 2011;168:1129-35. 
24. Ogawa K, Murota K, Shimura H, Furuya M, Togawa Y, Matsumura T, Masuta C. Evidence of capsaicin synthase activity of the Pun1-encoded protein and its role as a determinant of capsaicinoid accumulation in pepper. BMC Plant Biol. 2015;15:93.
25. Fu DQ, Zhu BZ, Zhu HL, Zhang HX, Xie YH, Jiang WB, Zhao XD, Luo KB. Enhancement of virus-induced gene silencing in tomato by low temperature and low humidity. Mol Cells. 2006;21:153-60.
Submit your next manuscript to BioMed Central and we will help you at every step:

- We accept pre-submission inquiries

- Our selector tool helps you to find the most relevant journal

- We provide round the clock customer support

- Convenient online submission

- Thorough peer review

- Inclusion in PubMed and all major indexing services

- Maximum visibility for your research

Submit your manuscript at www.biomedcentral com/submit
() Biomed Central 